\section{耍 Heighten Science \\ P U B L I C I T I O N S Corporation ISSN 2573-1726}

\title{
Parents Take-On Concussion: Advances in Sideline Research and Culture in Youth Sports
}

\author{
Arlene Silverio ${ }^{1,2}$, Scott Briggs ${ }^{3}$, Lisena Hasanaj ${ }^{4}$, J effrey Hurd, J r ${ }^{5}$, \\ Max Lahn ${ }^{6}$, Liliana Serrano ${ }^{4}$, Lucy Cobbs ${ }^{4}$, J enelle Raynowska ${ }^{4}$, \\ Rachel Nolan ${ }^{4}$, J oel Birkemeier ${ }^{7}$, Dennis Cardone ${ }^{8}$, Steven L. Galetta ${ }^{4,9}$ and \\ Laura J . Balcer ${ }^{4,7,9 *}$ \\ 'Division of Pediatric Emergency Medicine, Cohen Children's Medical Center of New York, \\ Hofstra North Shore LIJ School of Medicine, Hempstead, NY USA \\ ${ }^{2}$ Department of Emergency Medicine, New York University School of Medicine, New York, NY, \\ USA \\ ${ }^{3}$ OMS-II, Touro College of Osteopathic Medicine, New York, NY, USA \\ ${ }^{4}$ Department of Neurology, New York University School of Medicine, New York, NY, USA \\ ${ }^{5}$ Cornell University, Ithaca, NY, USA \\ ${ }^{6}$ Brown University, Providence, RI, USA \\ ${ }^{7}$ Department of Population Health, New York University School of Medicine, New York, NY, USA \\ ${ }^{8}$ Department of Orthopedics, New York University School of Medicine, New York, NY, USA \\ ${ }^{9}$ Department of Ophthalmology, New York University School of Medicine, New York, NY, USA
}

\begin{abstract}
*Address for Correspondence: Laura J. Balcer Department of Neurology, NYU School of Medicine, 240 East 38th Street, 20th Floor, New York, NY 10016. Tel: +1 646502 7681, Fax: + 1 212263 7721; Email: laura.balcer@nyumc.org

Submitted: 29 December 2016

Approved: 15 March 2017

Published: 16 March 2017

Copyright: $\circledast 2017$ Silverio A, et al. This is an open access article distributed under the Creative Commons Attribution License, which permits unrestricted use, distribution, and reproduction in any medium, provided the original work is properly cited.
\end{abstract}

Keywords: Concussion; Parents; Youth sports; Baseline testing; Sideline testing

\section{ABSTRACT}

Identifying concussion and initiating removal from play is challenging for even the most diligent youth sports organizations. Empowering parents to implement removal from play protocols and sideline testing may be the most practical plan at community levels to protect young athletes. We developed paradigms for community-based youth sports teams that incorporated both standard concussion protocols and research investigations. The research studies were designed to determine how sideline tests of vision, cognition and balance augment the capacity for parents and other responsible adults to identify youth athletes with concussion in ice hockey, football, lacrosse and cheerleading. Research-based sideline tests were performed at pre-season baseline sessions and during the season at the time of injury or as soon as symptoms were recognized by trained volunteer parent team testers. The combination of standard concussion protocols and research studies were performed for 510 athletes, aged 5-17 years, over 2.5 years through 5 athletic seasons. To implement the protocols and studies, approximately 80 student volunteers and parents were educated and trained on early concussion recognition and on baseline and sideline test administration. Over $80 \%$ of parent-identified head injuries were physician-confirmed concussions. Of the sideline tests performed, over two-thirds were administered within 24 hours of injury; the rest were performed within an average of 2.6 days post-injury since some athletes had delayed development of symptoms. Removal from play guidelines and standard concussion evaluation protocols were maintained in the context of the sideline testing research investigations. Based on this observational study, parents of youth athletes can be successfully empowered to perform rapid sideline tests in the context of existing concussion protocols. Implementation of objective testing may improve concussion identification and shift the culture of advocacy and responsibility towards parent groups to promote safety of young athletes. Ongoing investigations will further examine the impact of these programs on concussion management in youth sports.

\section{ABBREVIATIONS}

ACR: Advanced Concussion Recognition; CDC: Centers for Disease Control; IRB: Internal Review Board; NYS: New York State; NYU: New York University; SAC: 
Standardized Assessment of Concussion; SCAT3: Sports Concussion Assessment Tool$3^{\text {rd }}$ Edition; TBI: Traumatic Brain Injury

\section{INTRODUCTION}

Concussion is a mild form of traumatic brain injury (TBI), and is a major health concern in youth athletes. In the United States, there are an estimated 1.1 to 1.9 million sports- and recreation-related concussions among children $\leq 18$ years old [1]. Children and adolescents appear more vulnerable to concussion than older athletes [2,3], and even mild injuries can affect the thinking, learning, behavior, and emotions of a child's developing brain $[4,5]$.

Athletes who return to play before they have fully recovered are believed to be more susceptible to a subsequent concussion, prolonged recovery and potentially catastrophic consequences [6,7]. It is essential that concussions are recognized and diagnosed in a timely manner to ensure that youth athletes are treated appropriately and allowed to fully recover.

The prevalence of undiagnosed concussion remains an important problem in youth sports [1,8], in part because of reluctance or inability of young athletes to report symptoms [9-11]. Many elementary and high schools do not have the financial resources to have athletic trainers on the sidelines [12]. While coaches have increasingly received better education for determining whether youth athletes require further concussion evaluation [13], young athletes continue to play with symptoms, often without the awareness of coaches [14]. This may be due in part to a sports culture [15] that can conflate injuries with weakness, and the fact that coaches have many responsibilities during and after games.

One missing and vital piece of youth concussion assessment protocols may be the parents. Parents can help recognize a concussion during play and are also wellpositioned to notice symptoms after play (behavioral or emotional changes) that are not noted by the coach. However, many parents of youth athletes may not yet fully understand the significance of concussion [16-19].

A few youth sports programs have begun to examine the ability of parents to administer simple sideline tests; these tests have proven to be accurate in predicting concussion, and at minimum start conversations regarding injury [20-22]. This suggests that if parents are adequately informed on concussion protocols, they can be effectively empowered to assist with recognizing the signs and symptoms and to ensure that injured athletes are removed from play. The basis for creating the Advanced Concussion Recognition Initiative was to incorporate roles for parents in the context of both standard concussion protocols and investigative sideline testing. This represents a new strategy to share responsibility for reducing undiagnosed concussions and promoting youth safety in sports. The purpose of this "methods paper" is to describe in detail the protocols and procedures that youth teams and investigators used to empower parents for both concussion recognition and evaluation of sideline testing.

\section{MATERIALS AND METHODS}

Initiative aims and study overview

The specific aims of the Advanced Concussion Recognition (ACR) Initiative for the community youth sports groups were to (i) raise concussion awareness through education, (ii) develop a Head Injury Action Plan (Figure 1) applicable to all sports that prompts removal from play and incorporates the research sideline tests, (iii) train team parent volunteers on concussion recognition, administration of concussion screening tests and concussion first aid and (iv) provide baseline concussion screening for youth athletes. Concussion education, training and research studies of sideline baseline and 


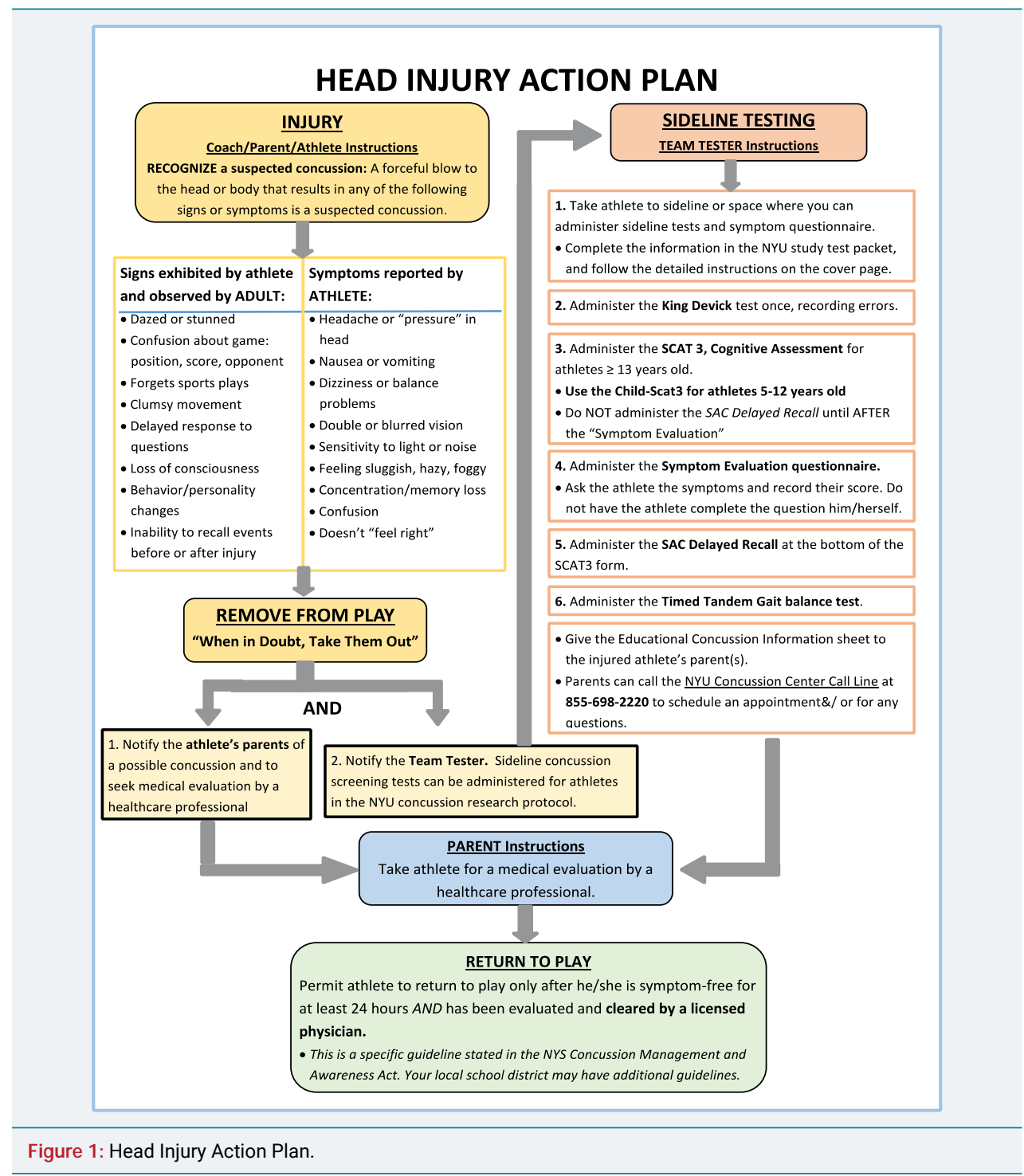

post-injury testing were conducted during five athletic seasons from September 2013 through March 2016 for four community youth sports organizations and one public school district. For the research studies, written informed consent and age-appropriate child assent were obtained for each youth sports participant. Informed consent and assent was a process administered by NYU investigators on-site for the youth leagues. All study protocols were approved by the NYU School of Medicine Institutional Review Board (IRB); participating youth sports organizations were required to submit a letter of participation from the organization's president or superintendent to the same IRB.

The ACR Initiative was designed and conducted to be integrated into the "realworld" environment of youth sports organizations, which necessarily incorporate parents, coaches and student volunteers. Research-based baseline and sideline tests included rapid number naming (King-Devick test), the Symptom Evaluation from the Sport Concussion Assessment Tool, 3rd Edition (SCAT3, for athletes 13 years and older) or Child-SCAT3 (for children 5-12 years old) and the timed tandem gait test. These testing components were selected based on research to date and anticipated ease of administration by lay people. All tests were repeated as soon as possible on the sidelines by trained parent testers in the event of a suspected concussion. The youth sports organizations included three suburban programs in ice hockey, lacrosse and football, one urban organization comprised of football and cheerleading, and a suburban public school district that included varsity and junior varsity teams in ice 
hockey, boys' and girls' lacrosse, and football. Major eligibility criteria included healthy youth and high school athletes aged 5-17 years who were registered athletes of the individual sports organization.

\section{Recruitment of youth organizations}

A grass roots recruitment strategy was employed in which participating athletes were recruited via email through the organizations' subscriber list, word-of mouth, onsite at various youth sports venues, as well as through various concussion educational presentations by NYU School of Medicine faculty and professional staff. For example, one particular presentation involved a movie screening at a local theater followed by a question and answer session involving concussion experts and members of the community.

\section{Advanced Concussion Recognition (ACR) program implementation}

\section{Stage 1: Planning}

Each youth sports organization identified a Concussion Coordinator to take the lead in executing the standard concussion protocols for the organization and coordinating with the NYU investigators and volunteer medical advisor supporting the community groups. The Concussion Coordinator then conducted preseason outreach via email to parents and coaches with information regarding the study protocol, as well as the dates, times and locations of the informed consent/ assent process and researchrelated pre-season administration of sideline testing. An online appointment sign-up for testing was created using a free web app during the third season to improve flow through the testing stations for participants in the research studies.

The Concussion Coordinator was responsible for recruiting parent volunteers to serve as each team's screening tester (aka the "Team Tester"). This individual received standardized training pre-season, assisted with the pre-season baseline testing sessions, administered the tests on the sideline and recorded the data in the event of an injury. To avoid conflicting interests and to allow coaches to continue managing the bench during a game or practice, coaches were not permitted to be Team Testers. All Team Testers were parents of participating athletes, and were present at most games or practices.

The Concussion Coordinator was also responsible for communicating with the coaches the standard concussion management protocol (implemented independently from the sideline testing performed for research purposes) and the importance of conducting sideline screening as soon as possible after injury.

For the pre-season baseline sessions for research-related testing, additional "person-power" was needed to test multiple teams in an efficient manner. Upper class high school students interested in obtaining community service hours were recruited, with one student trained by NYU investigators as the Student Coordinator. This student leader organized, enlisted and subsequently trained the high school student volunteers over a 2-year period to administer pre-season baseline tests. The Student Coordinator also worked with each organization's Concussion Coordinator to provide the necessary number of student volunteers at each of the pre-season baseline testing sessions. NYU investigators were onsite at every pre-season session to ensure that only athletes who had provided informed assent and parent/ guardian consent were included in the research-related sideline testing protocols, and that testing was performed according to specifications.

Finally, the Head Injury Action Plan was developed to incorporate the NYU study protocols investigating rapid sideline tests into each organization's existing standard concussion management guidelines as stated in the New York State (NYS) Concussion Management and Awareness Act [23]. The NYS Department of Health's Concussion 
Fact Sheet for Coaches and Sports Officials [24] was used as the standard guideline for parents and coaches and other responsible adults for concussion recognition and removal from play among all youth organizations.

\section{Stage 2: Education and training}

Concussion education as provided to parents and coaches included the basics of concussion, recognizing the signs and symptoms, and management (including removal from play). The potential utility of sideline screening assessments under research investigation, and instruction on administration, were also included. The Team Tester training sessions consisted of a didactic presentation followed by a small group handson workshop. Given that some parents could not make these sessions due to work and family conflicts, a "train-the-trainer" methodology was utilized, with the additional training provided by individuals who had previously completed training with NYU investigators including the community medical advisor, the youth organization's Concussion Coordinator or another experienced parent Team Tester.

\section{Stage 3: Pre-season baseline testing (research-based sideline tests)}

Pre-season testing was conducted over multiple sessions at various locations. At each session, informed consent and assent were obtained for interested/ willing participants by the NYU investigators. The Concussion Coordinator, Team Testers and student volunteers assisted with set up, check in, test administration, and clean up. Approximately 30-50 athletes were tested during the 1.5-2-hour sessions. Participants rotated through five stations (Figure 2) as described:

Station 1-Registration and Consent/ Assent: Parents of the youth athletes were required to attend the baseline testing session to sign informed consents; youth athletes signed assent forms as part of the consent/ assent process with NYU investigators. Parents/ guardians were then able to accompany their children through the stations and observe the testing. The voluntary nature of the research-based activities for the team concussion management protocols was emphasized as part of the consent/ assent process.

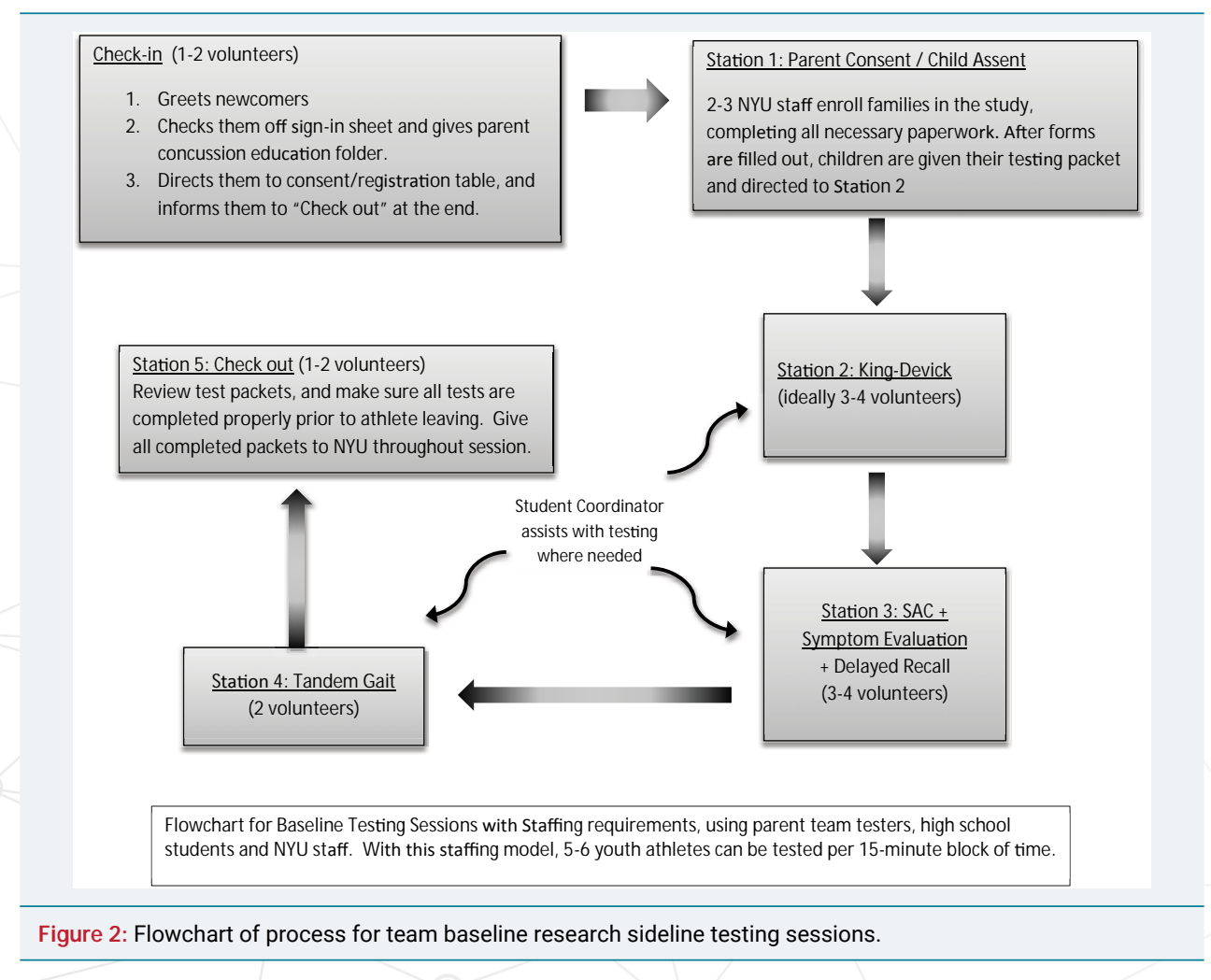


Station 2-King-Devick (K-D) Test of Rapid Number Naming: The K-D test is a vision-based rapid number naming assessment that takes less than 2 minutes to administer. Physical (spiral-bound notebook) versions of the test were provided by the manufacturers.

Station 3-SAC (Standard Assessment of Concussion) and Symptom Evaluation of the SCAT3 or Child-SCAT3: The SAC is a brief cognitive battery that captures the domains of orientation, immediate memory, concentration and delayed recall. A maximum total score of 30 is generated by adding the 4 sub scores: Orientation (maximum score=5), Immediate Memory (maximum score=15), Concentration (maximum score=5), and Delayed Recall (maximum=5). The SCAT3 Symptom Evaluation is a questionnaire that captures presence and severity of 22 symptoms associated with concussion.

Station 4-Timed Tandem Gait Test: The timed tandem gait test is a measure of balance and coordination in which athletes are timed in seconds on how quickly they can walk heel to toe along a 3-meter line back and forth. It is an integral part of the Child-SCAT3 for testing balance, and is feasibly administered particularly in the youth athlete population.

Station 5-Check Out: Concussion education handouts were distributed to participating athletes and their parents at the check-out station, and baseline testing and consent/ assent packets were reviewed for completeness of data.

\section{Stage 4: Post-injury sideline testing (research-based tests)}

During the athletic season, the teams were instructed to follow the Head Injury Action Plan in the event of a suspected concussion at a game or practice. A suspected concussion was defined as any forceful blow to the head or body that resulted in any of the signs or symptoms of concussion and were otherwise unexplained. Signs included any new neurological sign or symptom, such as but not limited to headache or pressure in the head, nausea, vomiting, dizziness, visual problems, sensitivity to light or noise, feeling foggy or hazy, difficulty with concentration or memory, confusion or not feeling right. In the case of a medical emergency, sideline testing was not performed and a 911 call was warranted with immediate transfer to the hospital. If the athlete associated with the research study did not require immediate transfer to a hospital and was physically able to perform the tests, the athlete was removed from play by a responsible adult, such as a coach, team manager, or parent and the Team Tester was notified to administer the sideline concussion tests.

Team Testers followed the detailed instructions provided in their testing packet for completing the data forms. They also provided the injured athlete's parents with the CDC Concussion Fact Sheet for Parents [25] as well as following basic concussion first aid of rest with limited physical and cognitive exertion until evaluated by a health care provider. All injured athletes, regardless of enrollment status in the research studies, were evaluated as per the standard concussion management protocols, removed from play and referred to a healthcare professional for formal medical diagnosis and care. In the event a concussion was not recognized at the time of injury and symptoms developed over a few hours or days, Team Testers were instructed to administer the sideline screening tests when feasible, even if days later, and to notify the investigators. The injured athlete's family was contacted within 24 hours for follow up.

\section{Stage 5: Post-season research data collection}

At the end of the athletic season, the numbers of injuries were obtained from the Concussion Coordinator for each organization, and the testing packets were collected. 


\section{RESULTS AND DISCUSSION}

There were 510 youth athletes enrolled in the sports of ice hockey, lacrosse, football and cheerleading enrolled as part of the NYU research protocols. Demographics for consented/ assented participants are presented in 1. In addition, there were 31 high school student volunteers, and approximately 50 parent volunteers trained as Team Testers among all the organizations. The student and parent volunteers performed 758 total pre-season baseline tests over 33 testing sessions throughout 5 athletic seasons; of note, many athletes participated in pre-season research-based sideline testing for more than one athletic season (thus the difference of 510 unique athletes vs. 758 preseason baseline tests).

Parents, coaches and athletes were encouraged, as part of the ACR initiative and by the standard concussion protocols, to identify any head injury and to initiate testing even if the injury was minor. As shown in table 1, there were a total of 75 reported head injuries identified by parents, coaches and athletes (including sports- and non-sportsrelated injuries). Of that number, $83 \%(n=62)$ were physician-confirmed concussions. Thus, in some cases where head injuries were identified by parents, coaches and athletes $(n=13)$, medical care was not sought because of early symptom resolution (within 24 hours, $n=12$ ) or the physician did not feel that a concussion had occurred $(n=1)$. There were 49 injuries for which sideline tests were administered in participating athletes. Thirty-seven athletes (76\%) had sideline tests administered within 24 hours of the injury, and $24 \%(n=12)$ were tested more than 24 hours later. This yielded an average injury-to-testing interval of 2.6 days; this finding was attributed to the fact that, for many youth athletes, symptoms developed over time and were sometimes not recognized immediately on the sideline. Of note, the parent Team Testers were not expected to make any determination of whether a concussion occurred; their role was to identify head injuries (in collaboration with the other parents, coaches and the athletes) and to perform the sideline testing for research purposes.

TheAdvanced Concussion Recognition(ACR) Initiative educates parents to recognize the signs and symptoms of concussion, provides guidelines to remove an athlete from

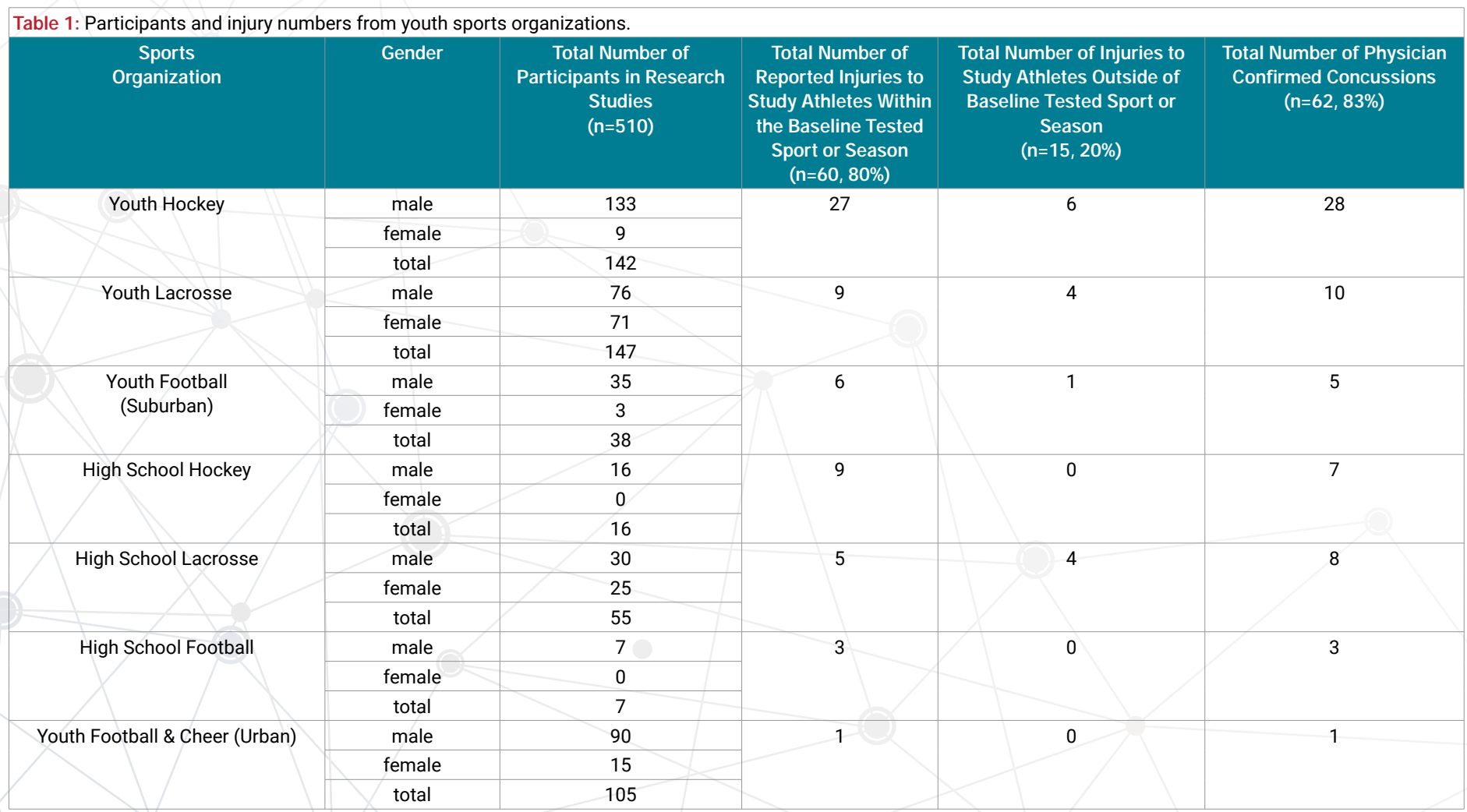


play, trains them to perform simple concussion screening tests, and institutes the basic first aid of concussion (i.e. rest) until an athlete can be evaluated by a healthcare provider. Experiences from this collaborative effort, which incorporated participation in sideline testing research with standard concussion protocols, demonstrate that implementing the ACR Initiative may be an effective strategy for improving concussion education, awareness, and management. The number of suspected concussions that were identified by parents and other adults using existing concussion protocols, and the percentage of those suspected concussions that were later confirmed by physician evaluation, indicate that parents are capable of learning and recognizing the signs and symptoms of concussion when provided with effective educational materials and instructions for implementing this knowledge. Furthermore, parents can be feasibly empowered to perform standardized, age-appropriate rapid sideline tests of vision, balance, cognition and symptoms.

Pre-season baseline as well as sideline data can be used to emphasize that comparing post-injury test results to pre-season scores is more effective than is the use of agebased norms. While normalized values are obtainable, this Initiative has demonstrated that pre-season baseline testing for sideline assessments can be performed accurately and effectively by students and parent Team Testers. Additionally, such testing is completed without many of the barriers created by requiring physicians or athletic trainers to be present for games or practice. Baseline and post-injury data from the research studies may offer more valuable information in understanding concussion recovery. Furthermore, pre-season baseline scores provide data that can account for the wide variability of individual scores that may be obtained in a youth cohort. The findings of the rapid sideline testing research have been published separately [26,27] and investigations are ongoing with new youth athlete cohorts to refine the numbers and to determine the value of a battery of rapid sideline tests.

While many school sports activities are subject to formal state concussion management laws and guidelines, a gap exists with respect to recognizing and managing concussions at the community level. At the same time, this is where the majority of youth sports activities occur. The ACR Initiative has the potential to make significant strides in filling this gap. A key element of the ACR Initiative is to educate parents to recognize concussion symptoms and to encourage the players and their families to seek appropriate medical attention. Leveraging research that shows the efficacy of multi-modal learning $[28,29]$, this initiative as reported for our organizations educated parents through verbal presentations, small group workshops, informational handouts and one-on-one trainings. The simplicity and ease of the test administration was important to examine as well. As such, parents became more comfortable administering the research-based sideline tests through practical experience. The ACR Initiative is also applicable to recognizing possible concussions and encouraging injured youth and their parents to seek appropriate medical attention in the context of all youth sports, across all age groups and in non-sports related situations (such as a bicycle accident or a fall at home or on the playground). Interestingly, while most injuries $(80 \%)$ occurred in the context of the sport for which an athlete was baseline tested for the research studies, $20 \%$ of the injuries occurred outside of the performance of sport, such as while at school. Thus, the universal applicability and sustainability of the ACR Initiative across all sports and recreational activities, as well as over time, was shown to be promising.

An important insight of this Initiative is that parents can play critical roles in concussion management that none of the other stakeholders can carry out as effectively. Coaches have multiple responsibilities that may make it difficult for them to recognize the symptoms of concussions and implement concussion protocols [30]. Having medically trained personnel at games or practices is impractical and prohibitively expensive at the youth level in many communities [31]. Given the unique 
relationships between parents/guardians and athletes, parents and guardians may be the ideal stakeholders to take responsibility for changing youth sports culture in relation to concussions. Parents and guardians are held accountable for their children's well-being and safety collectively; if they are provided with the appropriate tools and proper training, these adults can be empowered to take on a greater role in concussion recognition, diagnosis, and management in youth sports. Parents/guardians at the game or practice may perform rapid sideline tests much earlier than if the athlete were to wait to be tested until they were able to visit a doctor. Of course, such testing, as performed in the research studies herein, does not substitute for medical evaluation, but can serve to catalyze conversations about concussion and athlete safety at the time of the injury and lessen potential confusion among the responsible adults present. As such, sideline testing provides additional opportunities for Team Testers to reinforce to the parents/guardians of the injured athlete the importance of a physician evaluation.

The ACR Initiative also has the potential to greatly increase the concussion knowledge among the youth athletes themselves. Becoming familiar with the SCAT3/ Symptom Evaluation during the pre-season baseline session and volunteering to test other youth athletes may give young athletes a much better ability to recognize potential symptoms of concussion in themselves or among teammates. Along with coaches, and trainers, the youth athletes and parents can become an important part of a system that improves the recognition and treatment of concussion in youth sports. Stated differently, it does indeed "take a village."

The ACR Initiative can be implemented at very little cost to the youth sports organizations. The Initiative leverages the strong parent volunteer network that already exists in many communities, particularly with respect to youth sports teams. In our experience, parents want to be involved. The costs for testing equipment and licensing can be relatively minor even if borne by the youth sports organization, while the benefits can be enormous. In addition, rapid sideline tests designed to be nonproprietary may offer an alternative and are under investigation in youth, collegiate and professional athlete populations.

One of the potential issues with implementing ACR Initiative is that parents may attempt to interpret the results of the testing and make uninformed treatment decisions without consulting a physician. The intention of the ACR Initiative is of course not to replace the diagnosis by a physician, but to assist with recognition of potential concussions and institute removal from play for the safety of the athlete. Existing concussion protocols were followed, and the sideline tests in this case were performed for research purposes only. The Team Testers were only responsible for administering tests and collecting the data, and were instructed to refer all injured athletes to see a doctor for definitive diagnosis. The Team Tester education process should emphasize this point until such time as the sideline tests are considered standard for administration at baseline and at time of injury for aiding in identification of concussed athletes.

A key challenge in the evolution of the ACR Initiative was to create an injury action plan that is usable for parents across multiple sports, given the various national governing bodies, such as USA Hockey, US Lacrosse, and USA Football [32-34]. One approach may be to start with requirements mandated by state law and then identify commonalities that can be emphasized. A potential limitation of this study is that injured athletes were not referred to a single healthcare professional, but rather advised to see their personal physician. As such, the concussion diagnosis may differ based on the level of expertise of the evaluating provider.

\section{CONCLUSIONS}

Based on this observational study, there are multiple opportunities for further 
research with respect to the ACR Initiative. The concussion knowledge base of parents before and after the initiative can be evaluated in order to improve educational materials and ensure appropriate language readability for maximal effectiveness. Also, while the utility of the K-D test to identify concussion has been widely studied $[21,22,26,27,35,36]$, the reliability of the SAC, timed tandem gait, and Symptom Evaluation in youth athletes requires further study. Novel sideline tests for vision, balance and cognition may also emerge. Opportunities for additional research exist with respect to whether the implementation of the ACR Initiative leads to behavioral changes in coaches, athletes and parents in reporting and managing concussion, and in changing the culture for our youngest sports participants. Ongoing investigations will further examine the impact of these programs on concussion management in youth sports.

\section{ACKNOWLEDGEMENTS}

We thank the Concussion Coordinators, executive committee, coaches, team managers, and volunteers of the youth sports organizations and school administrators, staff and students for their contributions and collaboration. We are grateful to the families and children taking part in the research studies. Names of the specific organizations have been withheld to maximize confidentiality of the child participants in the research studies.

\section{REFERENCES}

1. Bryan MA, Rowhani-Rahbar A, Comstock RD, Rivara F, Seattle Sports Concussion Research Collaborative. Sports and recreation-related concussions in US youth. Pediatrics. 2016; 138. Ref.: https://goo.gl/pWaVrr

2. McCrory P, Collie A, Anderson V, Davis G. Can we manage sport related concussion in children the same as in adults? Br J of Sports Med. 2004; 38: 516-519. Ref.: https://goo.gl/bjsSFT

3. Sady MD, Vaughan CG, Gioia GA. School and the concussed youth: recommendations for concussion education and management. Phys Med Rehab Clin N Am. 2011; 22: 701-719. Ref.: https://goo.gl/NO3QBc

4. Carman AJ, Ferguson R, Cantu R, Comstock RD, Dacks PA, et al. Expert consensus document: mind the gapsadvancing research into short-term and long-term neuropsychological outcomes of youth sports-related concussions. Nat Rev Neurol. 2015; 11: 230-244. Ref.: https://goo.gl/XDI4DX

5. Centers for Disease Control and Prevention. Report to Congress on Traumatic Brain Injury in the US: Epidemiology and Rehabilitation. Atlanta, GA: National Center for Injury Prevention and Control; Division of Unintentional Injury Prevention, 2015.

6. Toledo E, Lebel A, Becerra L, Minster A, Linnman C, et al. The young brain and concussion: imaging as a biomarker for diagnosis and prognosis. Neurosci Biobehav Rev. 2012; 36: 1510-1531. Ref.: https://goo.gl/tE7xej

7. Rivara FP, Graham R. Sports-related concussions in Youth. JAMA. 2014; 311: 239-240. Ref.: https://goo.gl/PzOXiV

8. Meehan WP, Mannix RC, O'Brien MJ, Collins MW. The prevalence of undiagnosed concussions in athletes. Clin J of Sport Med. 2013; 23: 339-342. Ref.: https://goo.gl/61ayvM

9. McCrea M, Hammeke T, Olsen G, Leo P, Guskiewicz K. Unreported concussion in high school football players. Clin J of Sport Med. 2004; 14: 13-17. Ref.: https://goo.gl/hfxcy4

10. Chrisman SP, Quitiquit C, Rivara FP. Qualitative study of barriers to concussive symptom reporting in high school athletics. J Adolesc Health. 2013; 52: 330-335. Ref.: https://goo.gl/LcgvsZ

11. Kroshus E, Garnett B, Hawrilenko M, Baugh CM, Calzo JP. Concussion under-reporting and pressure from coaches, teammates, fans, and parents. Soc Sci Med. 2015; 134: 66-75. Ref.: https://goo.gl/aJYKyy

12. Pryor RR, Casa DJ, Vandermark LW, Stearns RL, Attanasio SM, et al. Athletic training services in public secondary schools: a benchmark study. J Athl Train. 2015; 50: 156-162. Ref.: https://goo.gl/Hwa8sU

13. Chrisman SP, Schiff MA, Chung SK, Herring SA, Rivara FP. Implementation of concussion legislation and extent of concussion education for athletes, parents, and coaches in Washington State. Am J Sports Med. 2014; 42: 11901196. Ref.: https://goo.gl/Tzdl9r

14. Rivara FP, Schiff MA, Chrisman SP, Chung SK, Ellenbogen RG, et al. The effect of coach education on reporting of concussions among high school athletes after passage of a concussion law. Am J Sports Med. 2014; 42: 11971203. Ref.: https://goo.gl/XRA32o 
15. Sports-Related Concussions in Youth: Improving the Science, Changing the Culture. Washington, DC: SportsRelated Concussions in Youth: Improving the Science. Changing the Culture. 2015; 180: 123-125. Ref.: https://goo.gl/ojzLyG

16. Coghlin CJ, Myles BD, Howitt SD. The ability of parents to accurately report concussion occurrence in their bantamaged minor hockey league children. J Can Chiropr Assoc. 2009; 53: 233-250. Ref.: https://goo.gl/YGUzWK

17. Bloodgood B, Inokuchi D, Shawver W, Olson K, Hoffman R, et al. Exploration of awareness, knowledge, and perceptions of traumatic brain injury among American youth athletes and their parents. J Adolesc Health. 2013; 53: 34-39. Ref.: https://goo.gl/028syZ

18. Lin AC, Salzman GA, Bachman SL, Burke RV, Zaslow T, et al. Assessment of parental knowledge and attitudes toward pediatric sports-related concussions. Sports Health. 2015; 7: 124-129. Ref.: https://goo.gl/VpGhgk

19. Sullivan SJ, Bourne L, Choie S, Eastwood B, Isbister S, et al. Understanding of sport concussion by the parents of young rugby players: a pilot study. Clin J Sport Med. 2009; 19: 228-230. Ref.: https://goo.gl/2VwEVQ

20. General Info on Concussion. Reston Raiders Hockey Club. 2016. Ref.: https://goo.gl/0eQBzf

21. Ventura RE, Jancuska JM, Balcer LJ, Galetta SL. Diagnostic tests for concussion. J Neuroophthalmol. 2015; 35 73-81. Ref.: https://goo.gl/2FDrSy

22. Leong DF, Balcer LJ, Galetta SL, Liu Z, Master CL. The King-Devick test as a concussion screening tool administered by sports parents. J Sports Med Phys Fitness. 2014; 54: 70-77. Ref.: https://goo.gl/GMxVIJ

23. The University of the State of New York. Guidelines for Concussion Management in the School Setting. 2016. Ref.: https://goo.gl/IQxNf6

24. New York State Department of Health. When in Doubt Take Them Out! Fact Sheet for Coaches and Sports Officials. 2016. Ref.: https://goo.gl/Uu3AS4

25. U.S. Department of Health and Human Services Centers for Disease Control and Prevention. Heads up Schools, A Fact Sheet for Parents. 2016. Ref.: https://goo.gl/wA1Dcw

26. Galetta KM, Morganroth J, Moehringer N, Mueller B, Hasanaj L, et al. Adding vision to concussion testing: a prospective study of sideline testing in youth and college athletes. J Neuroophthalmol. 2015; 35: 235-241. Ref.: https://goo.gl/htybOG

27. Galetta KM, Liu M, Leong DF, Ventura RE, Galetta SL, Balcer LJ. The King-Devick test of rapid number naming for concussion detection: meta-analysis and systematic review of the literature. Concussion. 2016; 1. Ref.: https://goo.gl/lcuq6y

28. Mrazik M, Dennison CR, Brooks BL, Yeates KO, Babul S, et al. A qualitative review of sports concussion education: prime time for evidence-based knowledge translation. Br J Sports Med. 2015; 49: 1548-1553. Ref.: https://goo.gl/nP7Pr5

29. Chrisman SP, Schiff MA, Chung SK, Herring SA, Rivara FP. Implementation of concussion legislation and extent of concussion education for athletes, parents, and coaches in Washington State. Am J Sports Med. 2014; 42: 1190 1196. Ref.: https://goo.gl/yYksMG

30. Newton JD, White PE, Ewing MT, Makdissi M, Davis GA, et al. Intention to use sport concussion guidelines among community-level coaches and sports trainers. J Sci Med Sport. 2014; 17: 469-473. Ref.: https://goo.gl/8Ek1WP

31. Tjoumakaris FP, Eck B, Freedman KB, Pepe MD, Austin L, et al. Cost benefit analysis of sports medicine team coverage: is it worth our while? Orthop J Sports Med. 2013; 1.

32. USA Hockey Concussion Management Program. USA Hockey. 2016. Ref.: https://goo.gl/dv0puj

33. US Lacrosse Concussion Management Plan Guidelines for U-19 Programs. US Lacrosse. 2016.

34. Youth Football Rules Book 2014. USA Football, Inc.

35. Galetta KM, Brandes LE, Maki K, Dziemianowicz MS, Laudano E, et al. The King-Devick test and sports-related concussion: study of a rapid visual screening tool in a collegiate cohort. J Neurol Sci. 2011; 309: 34-39. Ref. https://goo.gl/Rw9Fco

36. Galetta KM, Barrett J, Allen M, Madda F, Delicata D, et al. The King-Devick test as a determinant of head trauma and concussion in boxers and MMA fighters. Neurology. 2011; 76: 1456-1472. Ref.: https://goo.gl/pcvNIN 